\title{
Chronophin activation is necessary in Doxorubicin-induced actin cytoskeleton alteration
}

\author{
Su Jin Lee ${ }^{1}$, Jeen Woo Park ${ }^{1}$, Beom Sik Kang ${ }^{1}$, Dong-Seok Lee ${ }^{1}$, Hyun-Shik Lee ${ }^{1}$, Sooyoung Choi ${ }^{2}$ \& Oh-Shin Kwon ${ }^{1, *}$ \\ ${ }^{1}$ School of Life Science, College of Natural Science, Kyungpook National University, Daegu 41566, ${ }^{2}$ Department of Biomedical Sciences and \\ Research Institute for Bioscience and Biotechnology, Hallym University, Chunchon 24252, Korea
}

\begin{abstract}
Although doxorubicin (Dox)-induced oxidative stress is known to be associated with cytotoxicity, the precise mechanism remains unclear. Genotoxic stress not only generates free radicals, but also affects actin cytoskeleton stability. We showed that Dox-induced RhoA signaling stimulated actin cytoskeleton alterations, resulting in central stress fiber disruption at early time points and cell periphery cortical actin formation at a later stage, in HeLa cells. Interestingly, activation of a cofilin phosphatase, chronophin (CIN), was initially evoked by Dox-induced RhoA signaling, resulting in a rapid phosphorylated cofilin turnover leading to actin cytoskeleton remodeling. In addition, a novel interaction between $\mathrm{CIN}$ and 14-3-3 $\zeta$ was detected in the absence of Dox treatment. We demonstrated that $\mathrm{CIN}$ activity is quite contrary to $14-3-3 \zeta$ binding, and the interaction leads to enhanced phosphorylated cofilin levels. Therefore, initial CIN activation regulation could be critical in Dox-induced actin cytoskeleton remodeling through RhoA/cofilin signaling. [BMB Reports 2017; 50(6): 335-340]
\end{abstract}

\section{INTRODUCTION}

The cytotoxic property of doxorubicin has been widely used in anticancer therapy. The precise mechanism of Dox-induced cytotoxicity has not been fully explained. Cytotoxicity may occur via Dox intercalation into DNA and topoisomerase II inhibition leading to DNA damage. Reactive oxygen species (ROS) generation may also contribute to DNA damage, resulting in apoptotic cell death $(1,2)$. Dox also induces actin cytoskeleton altertions, largely through Rho family-dependent pathways (3). Rho family GTPases are key regulators of actin

${ }^{*}$ Corresponding author. Tel: +82-53-950-6356; Fax: +82-53-9432762; E-mail: oskwon@knu.ac.kr

https://doi.org/10.5483/BMBRep.2017.50.6.061

Received 12 April 2017, Revised 27 April 2017, Accepted 10 May 2017

Keywords: Actin cytoskeleton, Chronophin, Cofilin, Doxorubicin, RhoA signaling, 14-3-3 cytoskeletal dynamics in migratory cells, and are critically involved in cancer metastasis and angiogenesis (4). RhoA, Rac1, and Cdc42 contribute to cell motility by stimulating actin cytoskeleton rearrangements. Rac1 induces lamellipodial extensions at the leading edge of migratory cells (5), whereas Cdc42 is required for filopodia formation. RhoA is critical for actin stress fiber establishment and focal adhesion (6). Activated RhoA stimulates the downstream effector Rho-associated protein kinase (ROCK) and activates LIM kinase (LIMK), which phosphorylates cofilin. Phosphorylated cofilin (p-cofilin) is inactive for actin depolymerization, resulting in F-actin stabilization.

Cofilin is essential for actin filament elongation and remodeling. Precise spatial and temporal regulation of cofilindependent F-actin severing activity at the cell leading edge is crucial for cell motility (7). Rapid polymerization occurs at the newly generated barbed ends, allowing filament elongation. (8). Cofilin phosphorylation is catalyzed by LIMK and testicular kinase. P-cofilin is activated by dephosphorylation, facilitated by two cofilin-specific phosphatases, chronophin (CIN) and slingshot (SSH). CIN is a member of the haloacid dehydrogenase family of phosphatases; however, the molecular mechanisms of $\mathrm{CIN}$ regulation in actin dynamics are poorly characterized. Cofilin and actin depolymerizing factor (ADF) are specifically dephosphorylated by CIN and SSH. CIN also exhibits pyridoxal 5'-phosphate phosphatase activity (9), is involved in cell division (10), cofilin-actin rod formation in neurons (11), and chemotaxing leukocytes (12). PI3-kinase and phospholipase are also involved in signaling to p-cofilin dephosphorylation, which is directly linked to CIN regulation in vivo (13).

14-3-3 proteins also regulate actin dynamics by stabilizing p-cofilin. They interact with many proteins involved in actin cytoskeletal organization (14). 14-3-3 $\zeta$ overexpression is involved in pathways that directly contribute to cancer initiation

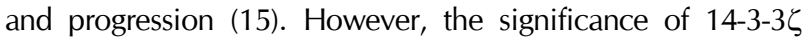
overexpression in many cancer types remains unknown.

We determined the effect of Dox on actin reorganization in Hela cells. CIN was identified as a key regulator in the Dox-induced cofilin regulatory system controlled by RhoA signaling. Activated CIN dephosphorylates p-cofilin and results in actin cytoskeleton rearrangement. Furthermore, we showed 
that $\mathrm{CIN}$ bound to $14-3-3 \zeta$, and this interaction was critical for regulating inhibition.

\section{RESULTS}

\section{Dox induces RhoA-GTP signaling}

RhoA-GTP signaling is involved in actin cytoskeleton organization and cell migration (16). If Dox-induced genotoxic stress is involved in RhoA signaling, Dox treatment may lead to cofilin phosphorylation modulation. To examine the morphological and cytoskeletal responses to genotoxic stress, we analyzed HeLa cells treated with Dox for 24 h (Fig. 1A). Dox treatment resulted in a clear development of thick actin fibers and cortical actin formation at the cell periphery. To explore the role of Dox in actin filament remodeling, we investigated the time course of p-cofilin levels (Fig. 1B). At early time points $(30 \mathrm{~min}), \mathrm{p}$-cofilin was gradually dephosphorylated by exposure to Dox. However, at later time points, p-cofilin dephosphorylation was inhibited, and p-cofilin started to accumulate. After $24 \mathrm{~h}$, we observed that p-cofilin level was higher than controls. To further examine whether Dox responses were involved in RhoA-GTP signaling, pull-down assays were performed for the time course of active RhoA detection. Indeed, similar to p-cofilin results, RhoA-GTP levels decreased at $30 \mathrm{~min}$ and increased until $24 \mathrm{~h}$ (Fig. 1C). Thus, Dox induced actin stress fiber formation through RhoA signaling, wherein $\mathrm{p}$-cofilin was negatively regulated at early time points.

\section{Dox stimulates p-cofilin dephosphorylation through CIN activation}

We investigated whether phosphatase activity was required for



Fig. 1. Time course of Dox-induced RhoA signaling. (A) Images of Alexa 555-phalloidin staining for F-actin of HeLa cells treated with or without Dox $(0.2 \mu \mathrm{g} / \mathrm{ml})$ for $24 \mathrm{~h}$. (B) Immunoblot of p-cofilin and cofilin in HeLa cell lysates treated with or without Dox for $10 \mathrm{~min}, 20 \mathrm{~min}, 30 \mathrm{~min}, 1 \mathrm{~h}, 16 \mathrm{~h}$, or $24 \mathrm{~h}$. Timedependent quantitative analysis showing p-cofilin reduction compared to that of total cofilin levels. (C) HeLa cells were treated with Dox for indicated times. Mean \pm SD $(n=3)$. ${ }^{* P}<0.05$.
Dox-induced p-cofilin dephosphorylation. p-cofilin was gradually dephosphorylated by exposure to Dox, while p-cofilin levels were essentially unchanged in presence of a phosphatase inhibitor, $1 \mathrm{mM}$ sodium vanadate (Fig. 2A). These results indicate the phosphatase involvement in this process. To identify specific phosphatases involved in vanadate-sensitive Dox-mediated p-cofilin dephosphorylation, we depleted CIN or SSH using siRNA (Fig. 2B). As expected, in control siRNA cells, the p-cofilin levels gradually decreased, and dephosphorylation occurred until 30 min. However, CIN knockdown led to p-cofilin accumulation without any significant decrease. Conversely, no significant differences in p-cofilin levels were detected in controls and in knock down SSH cells. This result suggests that Dox induces p-cofilin dephosphorylation through action of CIN in HeLa cells. To examine whether CIN had the same actin-dissolving or -disrupting effect on stress fibers, HeLa cells were incubated in presence or absence of CIN siRNA for $24 \mathrm{~h}$, followed by treatment with Dox for $30 \mathrm{~min}$.

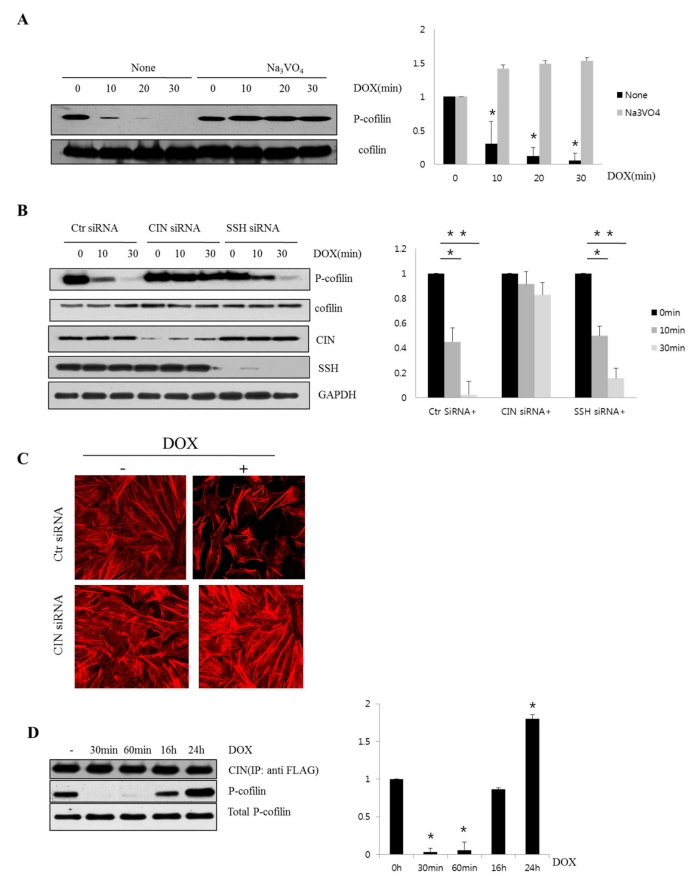

Fig. 2. CIN activation in Dox-induced p-cofilin dephosphorylation. (A) HeLa cells were pretreated with or without $0.1 \mathrm{mM} \mathrm{Na}_{3} \mathrm{VO}_{4}$ for $30 \mathrm{~min}$, and stimulated with Dox for 10,20 , or $30 \mathrm{~min}$. Cell lysates were subjected to immunoblot analysis with antibodies to p-cofilin and cofilin $(n=3, * \mathrm{P}<0.05)$. (B) HeLa cells were transfected with control, CIN, or SSH-1L siRNA for $24 \mathrm{~h}$, and stimulated with or without Dox for 10 or 30 min $(n=3$, *P < 0.05 and $* * \mathrm{P}<0.01)$. (C) HeLa cells were transfected with control or CIN siRNA for $24 \mathrm{~h}$, and stimulated with or without Dox for 30 min. (D) HeLa cells were transfected with FLAG-CIN stimulated without or with Dox for $30 \mathrm{~min}, 60 \mathrm{~min}, 16 \mathrm{~h}$, or 24 h. ${ }^{*} \mathrm{P}<0.05$ Time-dependent quantitative analysis of $\mathrm{p}$-cofilin was compared to that of CIN purified using anti-FLAG. 
Dox treatment resulted in cell flattening, decrease in cell size, and central stress fiber disruption, compared to control siRNA-transfected cells (Fig. 2C). In contrast, CIN siRNA cells exhibited minimal cell shape alteration and an increased number of cells with preserved central stress fibers. Thus, microscopic changes were no longer detected compared to non-treated control cells. These results indicated that $\mathrm{CIN}$ was involved in blocking formation of new stress fibers, and dissolving or disrupting existing stress fibers. In order to confirm CIN involvement in Dox-induced p-cofilin dephosphorylation, HeLa cells transfected with GFP-CIN were stimulated without or with Dox for up to $24 \mathrm{~h}$ (Fig. 2D). In vitro cofilin-phosphatase assays revealed that $\mathrm{CIN}$ activity was remarkably enhanced, and peaked at $30 \mathrm{~min}$, after which the p-cofilin levels gradually increased.

\section{CIN inhibition via 14-3-3 $\zeta$ binding}

HeLa cells transfected with GFP or GFP-CIN were lysed, and immunoprecipitation assay was carried out using anti-GFP antibodies (Fig. 3A). A protein of approximately $30 \mathrm{kDa}$, identified as $14-3-3 \zeta$ by mass spectrometric analysis (Fig. S1), was co-precipitated with CIN. FLAG-14-3-3 $\zeta$ was co-transfected in HeLa cells expressing GFP or GFP-CIN (Fig. 3B). FLAGtagged proteins were immunoprecipitated, and association between GFP-CIN and 14-3-3 $\zeta$ was confirmed by immunoblot analysis for CIN. Co-localization of $\mathrm{CIN}$ and endogenous $14-3-3 \zeta$ was also observed in the cytoplasm by immunofluorescence analysis (Fig. S2). 14-3-3 $\zeta$ interaction effects on



Fig. 3. $\mathrm{CIN}$ binding to $14-3-3 \zeta$. (A) HeLa cells were transfected with plasmids GFP (left lane) or GFP-CIN (right lane). Coimmunoprecipitation analyses were performed using GFP antibody.

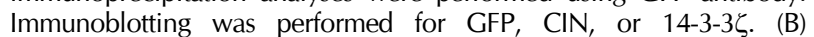
FLAG-14-3-3 $\zeta$ was co-transfected in cells expressing GFP alone (left lane) or GFP-CIN (right lane). Cell lysates were immunoprecipitated with anti-FLAG and immunoblotted for FLAG-tagged constructs or CIN. (C) GFP or GFP-CIN expressed in HeLa cells were purified using anti-GFP, and incubated with purified p-cofilin

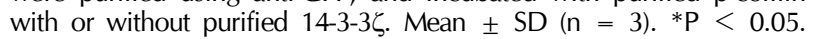

CIN phosphatase activity were investigated in vitro using a

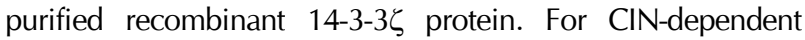
p-cofilin dephosphorylation assay, GFP or GFP-CIN was transiently transfected in cells for $24 \mathrm{~h}$, and GFP-tagged proteins were purified using anti-GFP. Precipitates were incubated with purified His tagged p-cofilin, with or without $14-3-3 \zeta$, in phosphatase buffer at $37^{\circ} \mathrm{C}$ for $1 \mathrm{~h}$. The reactions were stopped by addition of sample buffer and boiling, and p-cofilin levels were determined by immunoblot. We observed that p-cofilin levels decreased proportionally to CIN activity, but levels significantly increased in the presence of $14-3-3 \zeta$ (Fig. 3C). Thus, these results suggest that $14-3-3 \zeta$ inhibits CIN phosphatase activity in vitro.

\section{Dox-stimulated actin cytoskeleton alteration through $\mathrm{CIN}$ regulation}

If $\mathrm{CIN}$ depends on RhoA activation, CIN binding ability to $14-3-3 \zeta$ as well as cofilin phosphorylation should be inhibited by ROCK inhibitor Y-27632. To examine this hypothesis, HeLa cells were transfected with GFP-CIN treated with or without



Fig. 4. Dox-stimulated actin cytoskeleton alteration through regulation of ROCK and CIN. (A) HeLa cells co-transfected with GFP-CIN and FLAG-14-3-3 $\zeta$ were treated with or without Y-27632 for $30 \mathrm{~min}$. Cell lysates were used for immunoblot analysis with antibodies to p-cofilin (upper panel). In addition, co-immunoprecipitation was performed using GFP antibody, and immunoblotting

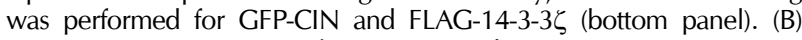

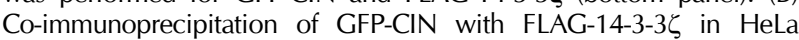
cells treated without or with Dox for 10 or 30 min. Graph depicting the amount of $14-3-3 \zeta$ and cofilin associated with CIN $(n=4, * P<0.05)$. (C) HeLa cells incubated for 30 min or $24 \mathrm{~h}$ in absence of Dox (upper panel). CIN protein was detected with an anti-CIN antibody, followed by detection with Alexa Fluor 488-conjugated goat anti-rabbit IgG (green). 
Y-27632 for 30 min (Fig. 4A). We analyzed the cell lysates by running western blot with p-cofilin antibodies (Fig. 4A, upper panel). Indeed, Y-27632 blocked the RhoA-dependent cofilin phosphorylation. In addition, cell lysates expressing GFP-CIN were immunoprecipitated and subjected to western blot analysis. Co-immunoprecipitation revealed that $\mathrm{CIN}$ bound to 14-3-3 $\zeta$ in absence of the inhibitor, whereas $14-3-3 \zeta$ binding levels were significantly reduced by Y-27632 treatment (Fig. $4 \mathrm{~A}$, bottom panel). These results suggest that $\mathrm{CIN}$ action on p-cofilin dephosphorylation is negatively regulated through 14-3-3 $\zeta$ binding in HeLa cells. To further explore the underlying mechanism of Dox-induced $\mathrm{CIN}$ activation, we assessed a time course of interaction between $\mathrm{CIN}$ and its negative regulator, 14-3-3 $\zeta$. GFP-CIN was transiently transfected into HeLa cells for $24 \mathrm{~h}$, and cells were treated with or without Dox for 10 or $30 \mathrm{~min}$. Cell lysates expressing GFP-CIN were immunoprecipitated and subjected to western blot analysis (Fig. 4B). 14-3-3 3 interaction with GFP-CIN time dependently decreased after Dox pretreatment, but cofilin binding levels were conversely increased. We examined the time course of morphological cytoskeleton responses to Dox treatment. Dox treatment after 30 min resulted in a slight reduction of actin cytoskeleton with central stress fiber disruption compared to control (Fig. 4C). In contrast, cells treated for $24 \mathrm{~h}$ with Dox showed a clear development of thick actin fibers at the cell periphery, and cortical contractile ring formation. CIN protein is primarily located in the cytoplasm. Immunofluorescence analysis showed that $\mathrm{CIN}$ was dispersed throughout the cytoplasm and was partially co-localized with the actin cytoskeleton. Following a 30 min or $24 \mathrm{~h}$ incubation in medium containing Dox, CIN clustered in the perinuclear region, and only limited co-localization with actin cytoskeleton was observed.

\section{DISCUSSION}

RhoA signaling regulates multiple biological processes such as cell cycling, apoptosis, and cell growth. Recent evidence suggests that Rho GTPase family members are key regulatory molecules of filamentous actin reorganization (17). Under cytotoxic stress conditions such as Dox cytotoxicity or serum starvation, Rho GTPase signaling cascade results in actin cytoskeleton remodeling (18). We investigated the mechanism in which the RhoA activity associated with Dox treatment was regulated in a time-dependent manner. Interestingly, apparent differences were observed in the temporal regulation of cofilin activation by Dox. At early time points, Dox promoted a rapid and transient decrease in p-cofilin levels as well as RhoA activity, with maximal inactivation occurring within $30 \mathrm{~min}$. The time courses were consistent with central actin fiber disassembling by Dox treatment. However, at later time points, both p-cofilin and RhoA activity gradually increased and returned to control levels after $60 \mathrm{~min}$. RhoA activity is regulated in a phasic manner for adhesion to fibronectin (19).
It is likely that RhoA is rapidly and transiently inhibited between 10 and 30 min when cells first bind fibronectin. In the second phase, signaling triggers stress fiber formation and focal adhesion via RhoA activation. Finally, RhoA activity gradually decreases to a level slightly higher than that of the initial inhibition. These data are consistent with our results. Thus, genotoxic stress stimulated central stress fiber disruption at early time points, thick actin fiber development, and cortical actin formation at the cell periphery. These data indicate that an early event in Dox-induced RhoA signaling is p-cofilin dephosphorylation, but subsequently cofilin activation is alleviated, and actin polymerization increases over time.

We searched for factors stimulating cofilin activity at initial time points, determining if any specific phosphatase was involved in this pathway. We first analyzed treatments with a general phosphatase inhibitor (Fig. 2A); next, we investigated through knockdown inhibition whether CIN was involved (Fig. 2B). As expected, p-cofilin dephosphorylation was the initial response of Dox cytotoxic stress. In addition, siRNA-mediated CIN knockdown resulted in limited changes in p-cofilin levels. Interestingly, siRNA-mediated SSH depletion attenuated p-cofilin dephosphorylation, similar to the siRNA control. Thus, CIN seems the most specific activator of cofilin in these stress conditions. These results indicate that $\mathrm{CIN}$ regulation is involved in RhoA signaling, and CIN activation, but not SSH, may be essential in regulating actin stability. Recently, similar results have been reported indicating that $\mathrm{CIN}$ depletion by siRNA-enhanced p-cofilin levels leads to a decrease in filament disassembly in epidermal growth factor-stimulated cells (20). $\mathrm{CIN}$ is redistributed temporally to the cell edge, where it regulates cofilin-dependent actin turnover and coordinates cell protrusion and retraction dynamics. In this study, similar to the temporal regulation of RhoA signaling, $\mathrm{CIN}$ was also activated initially and transiently after Dox treatment, thereby inducing central stress fiber disruption. Protease-activated receptor-2 (PAR-2) induces p-cofilin dephosphorylation through $\beta$-arrestin mechanisms consisting of LIMK activity inhibition and cofilin phosphatase activation (21). An initial event in PAR-2 signaling was $\mathrm{p}$-cofilin dephosphorylation and rephosphorylation over time, which is similar to what we demonstrated for Dox. Although it is not certain how CIN regulation is linked to lack of LIMK activity to cause increases in p-cofilin dephosphorylation, we confirmed that initial CIN activation via RhoA transient inhibitory regulation may be a key factor in p-cofilin turnover at early time points in Dox-induced HeLa cells.

Moreover, we also showed that $\mathrm{CIN}$ activity might be controlled by $14-3-3 \zeta$ binding, resulting in the control of actin cytoskeleton remodeling. In this work, direct interactions between $\mathrm{CIN}$ and 14-3-3 $\zeta$ in HeLa cells were demonstrated by reciprocal co-immunoprecipitations. $14-3-3 \zeta$ binds to key target proteins involved in regulating various cellular functions or subcellular localization. Like cofilin and SSH, LIMK and RhoA also bind to $14-3-3 \zeta$. In this study, the novel interaction between $\mathrm{CIN}$ and 14-3-3 $\zeta$ was confirmed biochemically. The 
involvement of interaction between $14-3-3 \zeta$ and $\mathrm{CIN}$ in cofilin activation was previously never explored. Interaction between $\mathrm{CIN}$ and $14-3-3 \zeta$ led to cofilin inactivation, whereas CIN was activated in absence of $14-3-3 \zeta$ and thereby reduced $p$-cofilin


complex were greatly attenuated with time, while cofilin levels were inversely increased (Fig. 4B). 14-3-3 $\zeta$ binding might stabilize CIN in the same way it binds and protects p-cofilin. Consistent with this observation, SSH binds $14-3-3 \zeta$, and this interaction inhibits the F-actin- mediated activation of cofilin-phosphatase activity (22). Thus, we conclude that CIN activation towards p-cofilin dephosphorylation and the interaction with 14-3-3 $\zeta$ are opposing.

We further examined the contribution of ROCK-dependent actin cytoskeleton remodeling. In RhoA-GTP signaling, RhoA interacts with ROCK, which phosphorylates and activates downstream targets, including LIMK. LIMK phosphorylates and inactivates ADF and cofilin, resulting in F-actin stabilization and stress fiber assembly (23). We showed that ROCK inactivation by Y-27632 resulted in cofilin activation (Fig. 4A). Simultaneously, CIN/14-3-3 complex formation was significantly attenuated, leading to dramatic CIN activation. These results indicate that the $\mathrm{CIN}$ and $14-3-3 \zeta$ inhibitory complex might be tightly associated with ROCK signaling. Moreover, Rac1 GTPase-regulated phosphatase disrupts SSH and 14-3-3 interaction to release catalytically active SSH (24). Thus, 14-3-3 $\zeta$ may be important not only for CIN activity regulation, but also for regulating actin polymerization through RhoA signaling. Further studies are required to elucidate the molecular mechanisms underlying $\mathrm{CIN}$ and $14-3-3 \zeta$ requirement in this pathway.

In summary, we have shown that Dox-induced RhoA signaling stimulates actin stress fibers through cytoskeleton remodeling. The finely regulated Rho signaling may initiate rapid p-cofilin dephosphorylation prior to actin stress fiber formation. As an early event, abnormal CIN activation causes p-cofilin turnover to cytoskeleton remodeling and central stress fiber disruption. Interestingly, CIN activity in cofilin activation is negatively regulated by $14-3-3 \zeta$ binding. To our knowledge, this is the first report on the involvement of $\mathrm{CIN}$ activation in response to Dox treatment, and CIN inactivation by binding of 14-3-35. Collectively, our data indicates that initial CIN activation regulation might be critical in Dox-induced actin cytoskeleton remodeling through ROCK/LIMK/cofilin pathways. This early actin depolymerization may be important in allowing later polymerization for cortical actin formation at the cell periphery.

\section{MATERIALS AND METHODS}

\section{Immunoprecipitation}

HeLa cells were grown in DMEM and transfection was performed with $1 \mu \mathrm{g}$ of DNA and $5 \mu \mathrm{l}$ of Lipofectamine (Invitrogen, Carlsbad, CA). Transfected cells were harvested after $24 \mathrm{~h}$ and homogenized in RIPA buffer containing protease inhibitors. Supernatants were incubated overnight with $3 \mu \mathrm{g}$ of either anti-GFP (Santa Cruz Biotechnology, Dallas, TX) or anti-FLAG antibody (Sigma-Aldrich, St. Louis, MO), followed by the addition of $100 \mu$ l Dynabeads-Protein-A (Thermo Fisher Scientific, Waltham, MA) conjugated slurry and incubation at $4^{\circ} \mathrm{C}$ for $1 \mathrm{~h}$. The immunoprecipitates were collected and washed three times with RIPA buffer.

\section{Immunoblot analysis}

Following antibodies were used: anti-CIN rabbit antibody (Cell Signaling Technology, Danvers, MA), anti-14-3-3 $\zeta$ (Santa Cruz Biotechnology), anti-p-cofilin (Ser3), anti-cofilin antibodies (Cell Signaling Technology), and anti-SSH1L antibody (Abcam, Cambridge, United Kingdom).

\section{CIN siRNA}

The CIN-specific siRNA sequences, 5'-GCGCCGUGCUUGUG GGCUA-3' (Oligo1) and 5'-GCACGCUUAUGGUGGGUGA3' (Oligo4) (25), were purchased from Dharmacon and used to transfect HeLa cells at a final concentration of $5 \mathrm{nM}$ in the presence of HiPerFect (Qiagen, Hilden, Germany).

\section{In vitro phosphatase assay}

The in vitro kinase reaction using $50 \mu \mathrm{M}$ ATP and purified cofilin $(0.2 \mathrm{mg} / \mathrm{ml})$ was performed as described (26). LIMK (Invitrogen) was incubated at $30^{\circ} \mathrm{C}$ for $1 \mathrm{~h}$, in $50 \mu$ of kinase buffer containing $2 \mu \mathrm{g}$ of purified cofilin and $25 \mu \mathrm{M}$ ATP. The reactions were terminated by the addition of SDS sample buffer.

\section{Measurement of Rho activity}

HeLa cells grown in DMEM were lysed in buffer containing 50 $\mathrm{mM}$ Tris (pH 7.2), $150 \mathrm{mM} \mathrm{NaCl}, 10 \mathrm{mM} \mathrm{MgCl}, 1 \mathrm{mM}$ PMSF, and a protease inhibitor cocktail. Lysates were incubated for $1 \mathrm{~h}$ at $4^{\circ} \mathrm{C}$ with glutathione agarose beads coupled to the Rhotekin Rho binding domain. Rho was detected by immunoblotting with anti-Rho-GTP and anti-RhoA antibodies. Data were standardized to the total RhoA content of each sample, and expressed as fold-change in RhoA activity (means \pm SD).

\section{Immunofluorescence}

Cells were fixed with $4 \%$ paraformaldehyde in PBS for $10 \mathrm{~min}$, washed three times with PBS, and permeabilized with $0.1 \%$ Triton X-100 in PBS for 15 min at room temperature. After washing three times in PBS, cells were blocked with $1 \%$ BSA for $1 \mathrm{~h}$ at room temperature. Incubation with anti-FLAG antibody was performed in blocking solution for $1 \mathrm{~h}$. Cells were simultaneously incubated with a secondary antibody and Alexafluor 555-conjugated phalloidin to examine the F-actin distribution.

\section{Statistical analysis}

Comparison between groups was performed using the Student's 
$t$ test. Data are means $\pm \mathrm{SD}$, and statistical significance is defined as $\mathrm{P}<0.05$.

\section{ACKNOWLEDGEMENTS}

This work was supported by Basic Science Research Program through the National Research Foundation of Korea (NRF) grant funded by the Ministry of Education (Grant No. 2014R1A1A2054781) and the Korea government (MSIP) (Grant No. NRF-2015R1A4A1042271).

\section{CONFLICTS OF INTEREST}

The authors have no conflicting financial interests.

\section{REFERENCES}

1. Pommier Y, Schwartz RE, Zwelling LA and Kohn KW (1985) Effects of DNA intercalating agents on topoisomerase II induced DNA strand cleavage in isolated mammalian cell nuclei. Biochemistry 24, 6406-6410

2. Branzei D and Foiani M (2008) Regulation of DNA repair throughout the cell cycle. Nat Rev Mol Cell Biol 9, 297308

3. Croft DR, Crighton D, Samuel MS et al (2011) p53mediated transcriptional regulation and activation of the actin cytoskeleton regulatory RhoC to LIMK2 signaling pathway promotes cell survival. Cell Res $21,666-682$

4. Ridley AJ (2015) Rho GTPase signalling in cell migration. Curr Opin Cell Biol 36, 103-112

5. Spiering D and Hodgson L (2011) Dynamics of the Rho-family small GTPases in actin regulation and motility. Cell Adh Migr 5, 170-180

6. Guo F, Debidda M, Yang L, Williams DA and Zheng Y (2006) Genetic deletion of Rac1 GTPase reveals its critical role in actin stress fiber formation and focal adhesion complex assembly. J Biol Chem 281, 18652-18659

7. Garg P, Verma R, Cook L et al (2010) Actin-depolymerizing Factor Cofilin-1 Is Necessary in Maintaining Mature Podocyte Architecture. J Biol Chem 285, 22676-22688

8. Bamburg JR (1999) Proteins of the ADF/cofilin family: Essential regulators of actin dynamics. Annu Rev Cell Dev Biol 15, 185-230

9. Huang TY, DerMardirossian C and Bokoch GM (2006) Cofilin phosphatases and regulation of actin dynamics. Curr Opin Cell Biol 18, 26-31

10. Jang YM, Kim DW, Kang TC et al (2003) Human pyridoxal phosphatase - Molecular cloning, functional expression, and tissue distribution. J Biol Chem 278, 50040-50046

11. Huang TY, Minamide LS, Bamburg JR and Bokoch GM (2008) Chronophin Mediates an ATP-Sensing Mechanism for Cofilin Dephosphorylation and Neuronal Cofilin-Actin Rod Formation. Dev Cell 15, 691-703
12. Delorme-Walker V, Seo JY, Gohla A, Fowler B, Bohl B and DerMardirossian C (2015) Chronophin coordinates cell leading edge dynamics by controlling active cofilin levels. Proc Nat Acad Sci U S A 112, E5150-E5159

13. Mouneimne G, Soon L, DesMarais V et al (2004) Phospholipase $C$ and cofilin are required for carcinoma cell directionality in response to EGF stimulation. J Cell Biol 166, 697-708

14. Jin J, Smith FD, Stark C et al (2004) Proteomic, functional, and domain-based analysis of in vivo 14-3-3 binding proteins involved in cytoskeletal regulation and cellular organization. Curr Biol 14, 1436-1450

15. Neal CL and Yu D (2010) 14-3-3zeta as a prognostic marker and therapeutic target for cancer. Expert Opin Ther Targets 14, 1343-1354

16. Raftopoulou $M$ and Hall A (2004) Cell migration: Rho GTPases lead the way. Dev Biol 265, 23-32

17. Vega FM and Ridley AJ (2008) Rho GTPases in cancer cell biology. Febs Letters 582, 2093-2101

18. Hartmann S, Ridley AJ and Lutz S (2015) The Function of Rho-Associated Kinases ROCK1 and ROCK2 in the Pathogenesis of Cardiovascular Disease. Frontiers in Pharmacology 6, 276-292

19. Arthur WT and Burridge $K$ (2001) RhoA inactivation by p190RhoGAP regulates cell spreading and migration by promoting membrane protrusion and polarity. Mol Biol Cell 12, 2711-2720

20. Neal CL and Yu DH (2010) 14-3-3 zeta as a prognostic marker and therapeutic target for cancer. Expert Opinion on Therapeutic Targets 14, 1343-1354

21. Zoudilova M, Min J, Richards HL, Carter D, Huang T and DeFea KA (2010) beta-Arrestins scaffold cofilin with chronophin to direct localized actin filament severing and membrane protrusions downstream of protease-activated receptor-2. J Biol Chem 285, 14318-14329

22. Nagata-Ohashi K, Ohta Y, Goto K et al (2004) A pathway of neuregulin-induced activation of cofilin-phosphatase Slingshot and cofilin in lamellipodia. J Cell Biol 165, 465-471

23. Wiggan O, Shaw AE, DeLuca JG and Bamburg JR (2012) ADF/cofilin regulates actomyosin assembly through competitive inhibition of myosin II binding to F-actin. Dev Cell 22, 530-543

24. Kligys K, Claiborne JN, DeBiase PJ et al (2007) The slingshot family of phosphatases mediates Rac1 regulation of cofilin phosphorylation, laminin-332 organization, and motility behavior of keratinocytes. J Biol Chem 282, 32520-32528

25. Delorme-Walker V, Seo JY, Gohla A, Fowler B, Bohl B and DerMardirossian C (2015) Chronophin coordinates cell leading edge dynamics by controlling active cofilin levels. Proc Natl Acad Sci U S A 112, E5150-5159

26. Okano I, Hiraoka J, Otera $\mathrm{H}$ et al (1995) Identification and characterization of a novel family of serine/threonine kinases containing two N-terminal LIM motifs. J Biol Chem 270, 31321-31330 